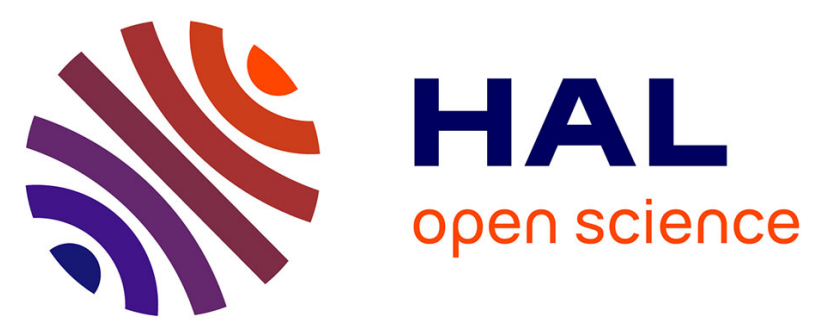

\title{
Evaluation and improvement of IEC 60891 correction methods for I-V curves of defective photovoltaic panels
}

Baojie Li, Anne Migan-Dubois, Claude Delpha, Demba Diallo

\section{To cite this version:}

Baojie Li, Anne Migan-Dubois, Claude Delpha, Demba Diallo. Evaluation and improvement of IEC 60891 correction methods for I-V curves of defective photovoltaic panels. Solar Energy, 2021, 216, pp.225-237. 10.1016/j.solener.2021.01.010 . hal-03237596

\section{HAL Id: hal-03237596 \\ https://hal-centralesupelec.archives-ouvertes.fr/hal-03237596}

Submitted on 4 Nov 2021

HAL is a multi-disciplinary open access archive for the deposit and dissemination of scientific research documents, whether they are published or not. The documents may come from teaching and research institutions in France or abroad, or from public or private research centers.
L'archive ouverte pluridisciplinaire HAL, est destinée au dépôt et à la diffusion de documents scientifiques de niveau recherche, publiés ou non, émanant des établissements d'enseignement et de recherche français ou étrangers, des laboratoires publics ou privés. 


\title{
Evaluation and Improvement of IEC 60891 Correction Methods for I-
} V Curves of Defective Photovoltaic Panels

\author{
Baojie LI ${ }^{1,2}$, Anne MIGAN-DUBOIS ${ }^{1}$, Claude DELPHA ${ }^{2}$, Demba DIALLO $^{1 *}$, \\ ${ }^{1}$ Université Paris-Saclay, CentraleSupélec, CNRS, GeePs, Sorbonne Université, 3-11 Rue Joliot Curie, Gif Sur Yvette, 91192, France \\ ${ }^{2}$ Université Paris-Saclay, CNRS, CentraleSupélec, L2S, 3 Rue Joliot Curie, Gif Sur Yvette, 91192, France \\ * Corresponding author: demba.diallo@ centralesupelec.fr
}

\begin{abstract}
Correction of the I-V curve permits the comparison of curves measured under different conditions for photovoltaic (PV) panels' health monitoring purpose. IEC 60891 has defined three standard procedures named 1, 2 and 3 for the correction. They were initially designed to correct the curves of healthy PV panels. However, their performance, when applied to I-V curves measured on faulty panels, is rarely discussed. This work evaluates these correction methods on I-V curves simulated under different environmental conditions and for five types of defects of varying severity. The results show that procedure 3 has a relatively better overall performance but is not suitable for rapid application in the field as it requires the determination of reference curves. It is found that procedures 1 and 2 could introduce distortion of the curve's shape, with a relative error of up to $13.8 \%$ and $6.4 \%$, respectively. A misestimation of $9.1 \%$ for key parameters of the curve has been observed, when using procedure 2 for maximum power. Based on the performance analysis, a new correction method is proposed to fit the corrected voltage. It can reduce the curve's average correction error by $31.3 \%$ compared to the original single curve correction method. Challenges and directions for future work are also presented.
\end{abstract}

Key words: Photovoltaic; I-V curve; I-V curve correction; IEC 60891; Fault detection and diagnosis;

\begin{tabular}{|c|c|c|c|}
\hline \multicolumn{4}{|c|}{ Nomenclature } \\
\hline$a$ & Irradiance correction factor & $V_{o c}^{\text {real }}$ & $V_{O C}$ of real curve at $\mathrm{STC}(\mathrm{V})$ \\
\hline$\alpha$ & Absolute temperature coefficient of $I_{S C}\left(\mathrm{~A} /{ }^{\circ} \mathrm{C}\right)$ & $V_{r p}$ & Voltage of inflexion point under PS (V) \\
\hline$\alpha_{r e l}$ & Relative temperature coefficient of $I_{S C}\left(\% /{ }^{\circ} \mathrm{C}\right)$ & PS & Partial shading \\
\hline$\beta$ & Absolute temperature coefficient of $V_{O C}\left(\mathrm{~V} /{ }^{\circ} \mathrm{C}\right)$ & $P_{m}$ & Maximum Power $(\mathrm{W})$ \\
\hline$\beta_{\text {rel }}$ & Relative temperature coefficient of $V_{O C}\left(\% /{ }^{\circ} \mathrm{C}\right)$ & $\mathrm{PV}$ & Photovoltaic \\
\hline $\mathrm{CE}$ & Curve error $(\%)$ & $\mathrm{OC}$ & Open circuit \\
\hline$G$ & Global in-plane irradiance $\left(\mathrm{W} / \mathrm{m}^{2}\right)$ & $R E$ & Relative error $(\%)$ \\
\hline GainPS & Block gain used in simulation to control PS degree & $R_{O C}$ & Resistance used in simulation for OC $(\Omega)$ \\
\hline$I$ & Current (A) & $R_{S}$ & Series resistance $(\Omega)$ \\
\hline & Current of corrected curve (A) & $R_{S \text { degra }}$ & Resistance used in simulation for Rs \\
\hline$I^{\text {real }}$ & Current of real curve at STC (A) & $S_{-}$_aegra & degradation $(\Omega)$ \\
\hline$I_{M P P}$ & Current at MPP (A) & $R_{S C}$ & Resistance used in simulation for SC $(\Omega)$ \\
\hline$I_{r p}$ & Current of inflexion point under PS (A) & $R_{s h}$ & Shunt resistance $(\Omega)$ \\
\hline $\begin{array}{l}I_{S C} \\
I_{S C}^{r e a l}\end{array}$ & $\begin{array}{l}\text { Short-circuit current (A) } \\
I_{S C} \text { of real curve at STC (A) }\end{array}$ & $R_{\text {sh_degra }}$ & $\begin{array}{l}\text { Resistance used in simulation for Rsh } \\
\text { degradation }(\Omega)\end{array}$ \\
\hline I-V curve & Current-voltage characteristics & $\mathrm{SC}$ & Short circuit \\
\hline MPP & Maximum power point & STC & Standard test condition \\
\hline$\kappa$ & Curve correction factor & TC & Temperature coefficient \\
\hline$V$ & Voltage (V) & $T m$ & Module temperature $\left({ }^{\circ} \mathrm{C}\right)$ \\
\hline$V_{M P P}$ & Voltage at MPP (V) & $\gamma$ & Interpolation constant for correction method \\
\hline$V_{O C}$ & Open-circuit voltage $(\mathrm{V})$ & & M3 \\
\hline$V_{O C}^{S T C}$ & $\begin{array}{l}V_{O C} \text { under STC in healthy condition (provided in } \\
\text { datasheet) }(\mathrm{V})\end{array}$ & & \\
\hline
\end{tabular}

\section{Introduction}

The current-voltage characteristics (I-V curves) measured from a faulty photovoltaic (PV) module or array (from now on, termed as faulty I-V curve) contain valuable information on the health status (Fadhel et al., 2019; Li et al., 2019). For a single module or small-scale PV string, one I-V tracer device can measure I-V curves under various meteorological conditions (Sarikh et al., 2020; Zhu and Xiao, 2020). Hardware solutions (integrated at inverter level) (Spataru et al., 2015) support are now commercially available to measure I-V curves at array or power plant level (Huawei, 2020). Therefore, with the increasing availability of field-measured I-V curves, I-V curves for PV health monitoring are becoming a hot topic (Mellit et al., 2018; Pillai and Rajasekar, 2018). 
Based on a field-measured I-V curve under any environmental conditions, there are two common ways (Mellit et al., 2018;

- Model-based difference analysis: Analyze the residue between the measured I-V curve (or the extracted features) and the simulated ones for identical environmental condition,

- Correction-based analysis: Correct the whole measured I-V curve (or the extracted features) to specific environmental condition (generally Standard Test Condition (STC) $\left(1000 \mathrm{~W} / \mathrm{m}^{2}\right.$ and $\left.25^{\circ} \mathrm{C}\right)$ ).

It is clear that for a model-based difference analysis, it is unnecessary to correct the I-V curve, but a suitable and accurate $\mathrm{PV}$ model is required.

However, for correction-based analysis, correction of the I-V curve is an indispensable step to make curves measured under different environmental conditions comparable. Based on the corrected curves, the open-circuit voltage $\left(V_{O C}\right)$, shortcircuit current $\left(I_{S C}\right)$, the voltage at the point of maximum power $\left(V_{M P P}\right)$, current $\left(I_{M P P}\right)$ and power $\left(P_{m}\right)$ are commonly extracted characteristics for fault diagnosis (Agrawal et al., 2020; Chen et al., 2017; Huang et al., 2019) or power loss analysis (Wang et al., 2020). Besides, equivalent series resistance $\left(R_{s}\right)$ or shunt resistance $\left(R_{s h}\right)$ are also commonly calculated from corrected curves to analyze the degradation mode of PV networks (Bouaichi et al., 2019; Silva et al., 2019).

In this sense, if there are significant errors in the corrected curves or the corresponding characteristics, the subsequent PV diagnosis's accuracy will be severely compromised. Therefore, correction of I-V curves plays a vital role in PV health monitoring.

There are no specific methods proposed for the correction of faulty I-V curves to the best of our knowledge. Consequently, most researchers adopt the original or simplified IEC 60891 (IEC 60891, 2009) correction procedures (Golive et al., 2019; Rajput et al., 2016). For example, several authors have adopted the procedure 1 in IEC 60891 to correct I-V curves measured under partial shading (PS) (Dolara et al., 2013), potential induced degradation (PID) (Martínez-Moreno et al., 2018), dust soiling (Tanesab et al., 2017, 2015), or hot spot (Ma et al., 2019). Besides, procedure 2 of IEC 60891 is also used to correct the I-V curves' key parameters (Chen et al., 2017). These parameters can then be used as inputs of a classifier (e.g., artificial neural network (ANN)) to automatically identify the fault types (PS, open circuit (OC), short circuit (SC), or shunt resistance degradation). Similar applications can also be found in (Zhu et al., 2018).

It should be noted that these methods based on the IEC 60891 standard have been all initially designed for the correction of curves measured from healthy PV panels. Moreover, in literature, the suitability of these methods correction of the I-V curves of defective panels is rarely studied, let alone whether the defects' characteristics are distorted by the correction and thus lead to a diagnostic error. All these issues are decisive for the diagnosis of PV faults (Triki-Lahiani et al., 2018). Thus, this paper aims to first evaluate the IEC 60891 methods' performance for the correction of faulty panels I-V curves. Then, based on IEC 60891 methods' performance analysis, an improved correction method will be proposed and evaluated.

The outline of the paper is as follows. Section 2 introduces the methodology from the simulation of faulty I-V curves, introduces the correction methods and performance evaluation metrics. Sections 3 and 4 present the correction performance using the single or multiple-curves-based methods, respectively. The comparison of these methods and guidance on future work are discussed in Section 5. Section 6 concludes the paper.

\section{Methodology for the evaluation of correction methods}

Since our target is to evaluate the error caused by the correction methods, it is essential to avoid the effects of other factors, like the measurement uncertainty on irradiance $G$, module temperature $T m$ and I-V curve, which could be up to $5 \%$ (Friesen et al., 2018). Therefore, the curves to be corrected are generated in simulation rather than from field measurement to ensure all the correction formula parameters are real values, without any bias.

\subsection{Simulation of $I-V$ curves}

\subsubsection{Simulation model}

A PV array is modelled under Simulink ${ }^{\circledR}$ to address several fault types. The array consists of two strings in parallel, and each string of three mc-Si modules in series, as shown in Fig.1 (a). Each module comprises three strings of twenty cells and three bypass diodes, as shown in Fig.1 (b). Table 1 lists the detailed module parameters. 
(a)

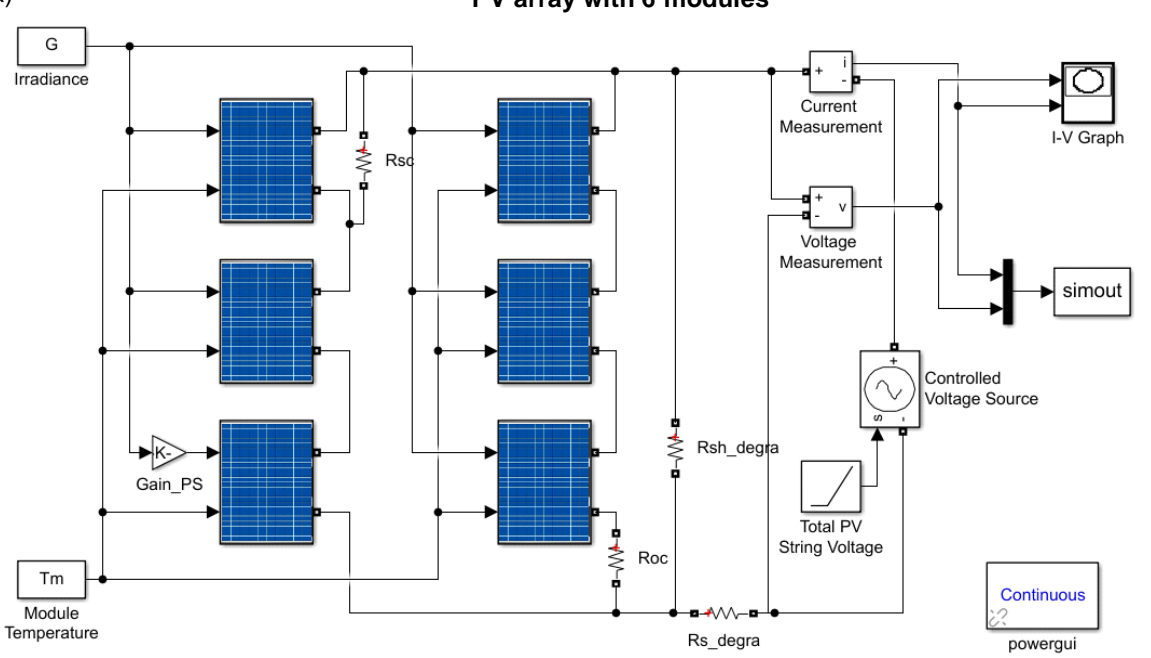

(b)

PV module

(3 diodes, 60cells)

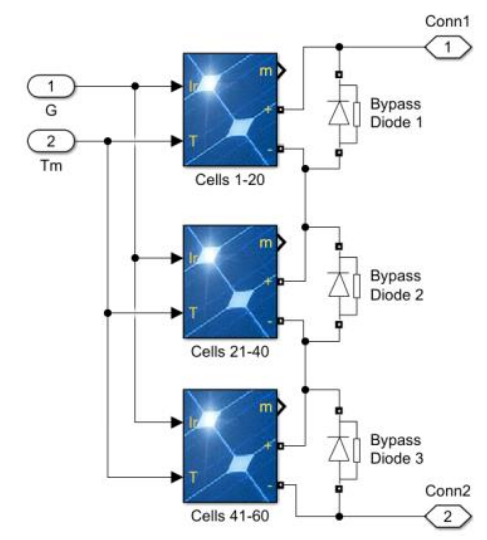

Fig. 1 Simulation model: (a) PV array structure, (b) PV module structure

Table 1 Parameter setting of mc-Si PV module

\begin{tabular}{lclc}
\hline \multicolumn{1}{c}{ Variable } & Value & \multicolumn{1}{c}{ Variable } & Value \\
\hline$I_{S C}$ & $8.64 \mathrm{~A}$ & $V_{M P P}$ & $31.80 \mathrm{~V}$ \\
$V_{O C}$ & $37.90 \mathrm{~V}$ & $\alpha_{\text {rel }}$ & $0.02 \% /{ }^{\circ} \mathrm{C}$ \\
$I_{M P P}$ & $6.52 \mathrm{~A}$ & $\beta_{\text {rel }}$ & $-0.36 \% /{ }^{\circ} \mathrm{C}$ \\
\hline
\end{tabular}

where, $\alpha_{\text {rel }}$ and $\beta_{\text {rel }}$ are the temperature coefficients (TC) of $I_{S C}$ and $V_{O C}$, respectively.

The characteristics of the array's I-V curve depend on:

- $G$ and Tm: the values are identical for all the modules and depend on the correction methodology, which will be detailed in Sections 3.1 and 4.1;

- Fault Parameters: the gain block and resistors added as shown in Figure 1 (a), which are detailed as follows.

\subsubsection{Configuration of faults}

With the gain block and the additional resistors, the model can simulate PV array under healthy and faulty conditions. In this work, five typical faults are considered:

1) Partial shading (PS): 1 module is shaded by adjusting the value of the GainPS coefficient which varies in the range $[0,1]$ and controls the irradiation of the module ;

2) Short-Circuit (SC): 1 module is short-circuited by connecting the resistance $R_{S C}$ in parallel;

3) Open-Circuit (OC): 1 string is open-circuited, by connecting the resistance $R_{O C}$ in series;

4) Rs degradation (Rs degra): the equivalent series resistance of the array is increased by putting in series additional resistance $R_{S_{-} \text {degra }}$;

5) Rsh degradation (Rsh degra): the equivalent shunt resistance of the array is decreased by connecting in parrallel the resistance $R_{\text {sh_degra }}$;

Table 2 summarizes the different fault parameters and their corresponding range of variation used to analyze the impact of fault severity on the correction performance, presented in sections 3.2 and 4.2.

Table 2 Parameter setting for the different conditions

\begin{tabular}{cccccc}
\hline Condition & GainPS & $R_{S C}(\Omega)$ & $R_{O C}(\Omega)$ & $R_{S_{-} \text {degra }}(\Omega)$ & $R_{\text {Sh_degra }}(\Omega)$ \\
\hline Healthy & 1 & $10^{5}$ & $10^{-5}$ & $10^{-5}$ & $10^{5}$ \\
PS 1 module & {$[\mathbf{0 - 1}]$} & $10^{5}$ & $10^{-5}$ & $10^{-5}$ & $10^{5}$ \\
SC 1 module & 1 & $\mathbf{1 0}^{-5}$ & $10^{-5}$ & $10^{-5}$ & $10^{5}$ \\
OC 1 string & 1 & $10^{5}$ & $\mathbf{1 0}^{5}$ & $10^{-5}$ & $10^{5}$ \\
Rs degradation & 1 & $10^{5}$ & $10^{-5}$ & {$\left[\mathbf{1 0}^{-5}-\mathbf{2}\right]$} & $10^{5}$ \\
Rsh degradation & 1 & $10^{5}$ & $10^{-5}$ & $10^{-5}$ & {$\left[\mathbf{1 0}^{5}-\mathbf{1 0}\right]$} \\
\hline
\end{tabular}




\subsection{Correction methods}

Through the literature review, the most common I-V curve correction methods are based on three procedures (hereafter, called M1, M2, and M3) proposed in IEC 60891 (IEC 60891, 2009). These methods and a proposed M2-based improved method are detailed as follows:

- Method 1 (M1):

$$
\begin{gathered}
I_{2}=I_{1}+I_{S C 1}\left(G_{2} / G_{1}-1\right)+\alpha\left(T_{m 2}-T_{m 1}\right) \\
V_{2}=V_{1}-R_{S}\left(I_{2}-I_{1}\right)-\kappa I_{2}\left(T_{m 2}-T_{m 1}\right)+\beta\left(T_{m 2}-T_{m 1}\right),
\end{gathered}
$$

where, $I_{1}$ and $I_{2}, V_{1}$ and $V_{2}, T_{m 1}$ and $T_{m 2}, G_{1}$ and $G_{2}$ are the current, voltage, module temperature, and irradiance before, and after correction, respectively; $I_{S C 1}$ is the short-circuit current before correction; $\alpha$ and $\beta$ are the PV module absolute TC of $I_{S C}$ and $V_{O C}$, respectively; $\alpha=\alpha_{r e l} \cdot I_{S C}^{S T C}, \beta=\beta_{r e l} \cdot V_{O C}^{S T C}, \alpha_{r e l}$ and $\beta_{r e l}$ are the relative TC of $I_{S C}$ and $V_{O C}$ (presented in Table 1); $R_{S}$ is the internal series resistance and $\kappa$ is the curve correction factor.

- Method 2 (M2):

$$
\begin{gathered}
I_{2}=I_{1}\left(1+\alpha_{r e l}\left(T_{m 2}-T_{m 1}\right)\right) G_{2} / G_{1} \\
V_{2}=V_{1}+V_{o c 1}\left[\beta_{r e l}\left(T_{m 2}-T_{m 1}\right)+a \cdot \ln \left(G_{2} / G_{1}\right)\right]-R_{s}\left(I_{2}-I_{1}\right)-\kappa \cdot I_{2}\left(T_{m 2}-T_{m 1}\right),
\end{gathered}
$$

where, $V_{O C 1}$ is the $V_{O C}$ before correction; $a$ is the irradiance correction factor; $R_{S}$ and $\kappa$ are determined by the procedure described in (IEC 60891, 2009).

The determination of the coefficients $a, R_{s}$ and $\kappa$ for M1 and M2 requires a group of I-V curves at the same $G$ or at the same $\mathrm{Tm}$, which is troublesome and time-consuming to fulfill in real life. Therefore, in most M1 and M2 applications, these coefficients are either tuned via simulation under healthy condition (Silva et al., 2019) or neglected (Dolara et al., 2013) but to the detriment of lower performance. Therefore, in this paper, these coefficients will not be neglected and determined following the standard routine, i.e., via the simulation under healthy condition.

- New Method 2 (NewM2):

In fact, as it will be shown later, M1 and M2 do not perform well under all the tested faulty conditions. Thus, a new method denoted NewM2 is proposed. It uses the same formula as M2 for current correction. But for the voltage correction, the term ' $V_{O C 1}$ ' in (4) is replaced by ' $V_{O C 1}\left[1+\beta_{\text {rel }}\left(25-T_{1}\right)\right]$ '. The reason for this change will be explained in Section 3.2.1 while analyzing the correction performance. Therefore, the equations for NewM2 are:

$$
\begin{gathered}
I_{2}=I_{1}\left(1+\alpha_{r e l}\left(T_{m 2}-T_{m 1}\right)\right) G_{2} / G_{1} \\
V_{2}=V_{1}+V_{O C 1}\left[\mathbf{1}+\boldsymbol{\beta}_{\text {rel }}\left(\mathbf{2 5}-\boldsymbol{T}_{\mathbf{1}}\right)\right]\left[\beta_{r e l}\left(T_{m 2}-T_{m 1}\right)+a \cdot \ln \left(G_{2} / G_{1}\right)\right]-R_{s}\left(I_{2}-I_{1}\right)-\kappa \cdot I_{2}\left(T_{m 2}-T_{m 1}\right)
\end{gathered}
$$

- Method 3 (M3):

Different from M1, M2 or the NewM2, M3 is free from correction coefficient but needs an interpolation constant $\gamma$ :

$$
\begin{gathered}
I_{3}=I_{1}+\gamma\left(I_{2}-I_{1}\right) \\
V_{3}=V_{1}+\gamma\left(V_{2}-V_{1}\right)
\end{gathered}
$$

According to (IEC 60891, 2009), M3 requires at least 2 reference curves to obtain one corrected curve at specified $G$ or Tm by calculating $\gamma$ using (7) or (8). Thus, to correct a curve with a requirement on both $\mathrm{G}$ and $T m$, three curves are necessary.

$$
\begin{aligned}
& G_{3}=G_{1}+\gamma\left(G_{2}-G_{1}\right) \\
& T_{3}=T_{1}+\gamma\left(T_{2}-T_{1}\right)
\end{aligned}
$$

In summary, the implementation of M1, M2, and NewM2 only requires one single I-V curve, while M3 requires multiple curves. Since their correction procedures are different, their evaluation performance will be conducted separately (in Sections 3 and Section 4, respectively). Since STC $\left(G=1000 \mathrm{~W} / \mathrm{m}^{2}\right.$ and $\left.T m=25^{\circ} \mathrm{C}\right)$ is more commonly adopted as the target condition for I-V curve-based diagnosis, in the following, if not stated, the correction of I-V curves refers to the correction to STC.

\subsection{Performance evaluation metrics}

The evaluation of correction performance will be conducted from two aspects, i.e., from the whole I-V curve and single extracted parameters (e.g., maximum power $P_{m}, V_{O C}$ and $I_{S C}$ ). Firstly, for the whole I-V curve, the curve error $(C E)$ is adopted as the metric, calculated by the normalized root-mean-square error between the corrected curve and the one 
simulated-at-STC (from now on called real curve). It should be noted that the real curve only means that $G$ and $T m$ are at STC, but the array condition could be either healthy or faulty.

$$
C E=\frac{\sqrt{\frac{1}{N} \sum_{i=1}^{N}\left(I_{i}^{c}-I_{i}^{\text {real }}\right)^{2}}}{I_{S C}^{\text {real }}} \times 100 \%
$$

where, $I_{i}^{c}$ and $I_{i}^{r e a l}$ are the current values from the corrected and actual curve, respectively, for the same voltage $V_{i} . \quad V_{i}$ is the $i^{\text {th }}$ element of the voltage vector consisting of $\mathrm{N}$ points linearly distributed in the interval between 0 and $V_{\max }$ with a constant step ( $V_{\max }$ is constant for all the conditions). The value of $V_{\max }$ can be set higher than Voc in order to prevent the voltage after correction from exceeding the set value. In this study, $V_{\max }$ is set as $120 \mathrm{~V}$ and $N$ at $100 . \quad I_{S C}^{\text {real }}$ refers to the $I_{S C}$ extracted from the real curve.

Then, regarding the parameters, the correction is evaluated with the relative error $(R E)$ :

$$
R E_{X}=\frac{X^{c}-X^{\text {real }}}{X^{\text {real }}} \times 100 \%
$$

where, $X^{c}$ and $X^{\text {real }}$ are the parameters from the corrected and real curve, respectively. $X$ can be a data coming directly from the curve (such as $P_{m}, V_{O C}$ or $\left.I_{S C}\right)$ or one of the parameters of the single-diode model $\left(R_{S}\right.$ or $R_{S h}$ for example) whose evolution can be considered as a signature of a defect.

\section{Performance of correction methods using single I-V curve}

This section focuses on the correction methods based on a single I-V curve, i.e., M1, M2 and NewM2. Firstly, the selection of $G$ and $T m$ to generate the curves is presented in Section 3.1. Then, the correction performance (using the metrics $C E$, and $R E$ ) will be evaluated using the curves simulated under constant or variable fault severity, presented in sections 3.2 and 3.3, respectively.

\subsection{Selection of $G$ and $T m$ based on field measurement}

To evaluate the correction methods with real environmental conditions, the selection of $G$ and $T m$ to generate the curves is based on field measurement (in SIRTA meteorological and climate observatory (Migan et al., 2015), France) of one mc-Si PV module (identical to those used in the simulation in Section 2.1.1) during summer as illustrated in Fig. 2. To minimize the correction error, commonly, the curves' irradiance for correction is selected in the highest range (Ding et al., 2014). Therefore, in our case studies, the lower bound of $G$ is set as $800 \mathrm{~W} / \mathrm{m}^{2}$.

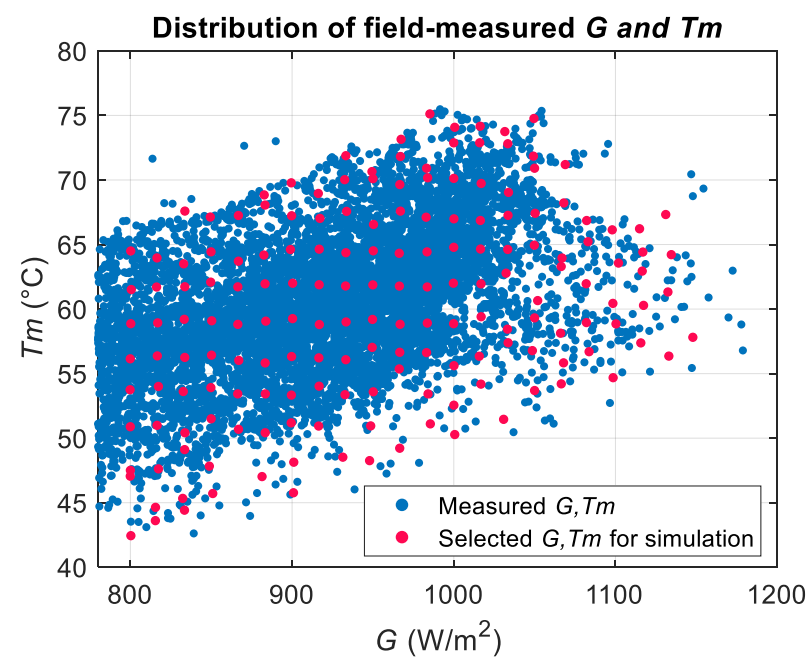

Fig. 2 Selected $G$ and $T m$ based on field-measurement during summer for one mc-Si PV module

In Fig. 2, the blue points represent the measured $G$ and $T m$, while the red ones are selected for the simulation. A group of 174 pairs of $G$ and $T m$ is selected based on a quasi-uniform distribution within the area enclosed by the blue points.

\subsection{Correction performance using curves for defects of constant severity}

The fault severity is firstly set constant to investigate the impact of the selected $G$ and $T m$ :

1) GainPS $=0.2$ for partial shading, 
2) Rs_degra $=1 \Omega$ for series resistance degradation,

3) Rsh_degra $=30 \Omega$ for shunt resistance degradation

Based on the generated curves, the correction performance can now be evaluated. Section 3.2.1 examines the shape of the corrected curves. Section 3.2.2 analyzes the performance evaluation metrics $C E$ and $R E$ for key curve parameters, and Section 3.2.3 studies the $R E$ for the fault features.

\subsubsection{Corrected I-V curve form}

Using M1, M2 and NewM2, the curves before and after correction are displayed in Fig. 3.
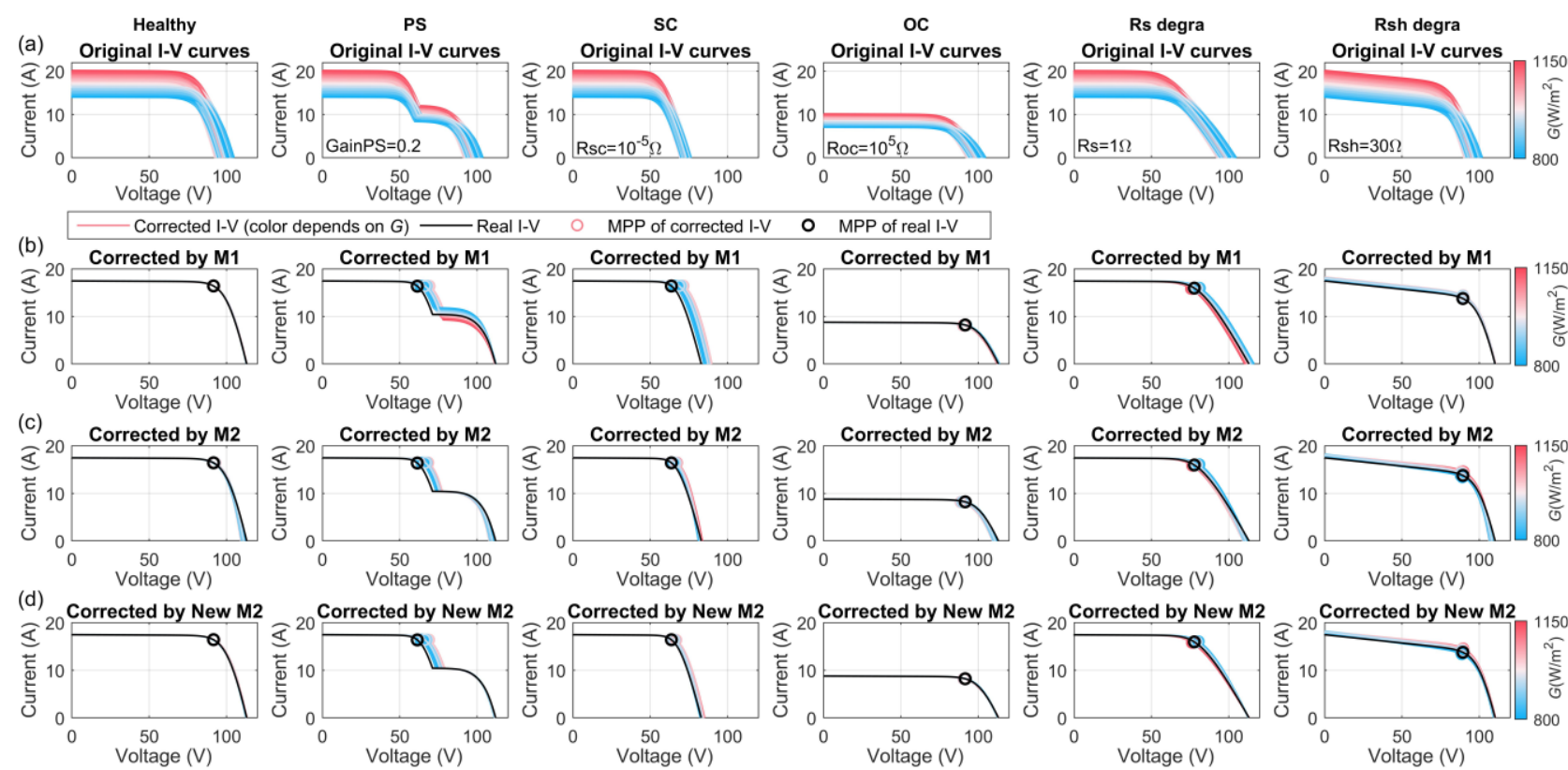

Fig. 3 Correction results, (a): curves simulated for correction (each condition contains 174 curves with field-measured combinations of $G$ and $T m$ at constant fault severity), (b): corrected curves using M1, (c): corrected curves using M2, (d): corrected curves using NewM2 (the displayed color of each curve is determined by the irradiance value with the colorbar on the right side of the figure, the circles on the curves represent the MPP)

For the corrected curves, apparent deviations to the real I-V curve can be observed for most faulty conditions using the three methods. These deviations are due to the combined effect of voltage and current corrections, reflected along the $x$ and $y$ axes, respectively. They are now analyzed as follows:

1) Voltage correction:

The deviations due to voltage corrections are observable in all the cases when using M2, but only for SC and Rs degradation when using M1, and for SC when using the NewM2. To analyze these results, we rearrange the voltage correction formulas (2), (4), and (6) as it follows:

$$
V_{2}=k_{1} \cdot V_{1}+k_{2} \cdot\left(T_{m_{2}}-T_{m 1}\right)+\mathrm{k}_{3} \cdot \ln \left(G_{2} / G_{1}\right)+k_{4} \cdot\left(I_{2}-I_{1}\right)+k_{5} \cdot I_{2} \cdot\left(T_{m_{2}}-T_{m 1}\right),
$$

where the different coefficients $k_{i}(i=1, \ldots, 5)$ for each method, are given in Table 3 .

Table 3 Coefficients for voltage correction in M1, M2 and NewM2

\begin{tabular}{|c|c|c|c|c|c|}
\hline Method & $k_{1}$ & $\boldsymbol{k}_{2}$ & $k_{3}$ & $k_{4}$ & $k_{5}$ \\
\hline M1 & 1 & $\beta_{\text {rel }} \cdot V_{O C}^{S T C}$ & 0 & $-R_{S}$ & $-k$ \\
\hline M2 & 1 & $\beta_{\text {rel }} \cdot \boldsymbol{V}_{\boldsymbol{O C} \mathbf{1}}$ & $a \cdot V_{O C 1}$ & $-R_{S}$ & $-k$ \\
\hline NewM2 & 1 & $\begin{array}{c}\beta_{r e l} \cdot V_{o c 1} \cdot \\
{\left[1+\beta_{r e l}\left(T_{m 2}^{*}-T_{m 1}\right)\right]}\end{array}$ & $a \cdot V_{O C 1}$ & $-R_{S}$ & $-k$ \\
\hline
\end{tabular}

Firstly, we compare M1 and M2. Based on the tuning procedure in the standard IEC 60891, $k_{4}$ and $k_{5}$ are similar for the two methods. As for $k_{3}$, it is 0 in M1. In M2, since both the coefficient $a$ and ' $\ln \left(G_{2} / G_{1}\right)$ ' are small values, their product is a second-order term, and its contribution is negligible (within $\pm 0.3 \%$ ). As a consequence, the main difference between $\mathrm{M} 1$ and M2 lies in $k_{2}$. It is noteworthy that $k_{2}$ for M1 is constant, while for M2, it depends on $V_{O C 1}$ ( $V_{O C}$ of the curve to correct). In Fig. 3 (b), there is almost no distortion of the corrected curves when using M1 under healthy condition, which demonstrates the voltage's correction efficiency. As for M2, since $T_{m}$ of the curves to correct $\left(T_{m 1}\right)$ are all higher than 
the target $T_{m}\left(T_{m 2}, 25^{\circ} \mathrm{C}\right), V_{o c 1}$ is thus always lower than $V_{O C}^{S T C}$ (impact of $G$ on $V_{O C 1}$ is negligible compared to $T m$ ). Accordingly, $k_{2} \cdot\left(T_{m 2}-T_{m 1}\right)$ in M2 is lower than in M1. Therefore, the corrected voltage in M2 is lower than in M1. That is the reason why in Fig. 3 (c), we can observe the slight distortions of the corrected curves shifted on the left side of the real curve round the open-circuit point.

Besides, the significant correction error under SC and PS for M1 and M2 also originates from the setting of $k_{2}$. For SC, to be specific, short-circuit is introduced in one module of one string. As a consequence, $V_{O C}^{r e a l}$ is different from $V_{O C}^{S T C}$ (value extracted from the datasheet, i.e., in healthy case), and is equal to around $2 / 3$ of $V_{O C}^{S T C}$. Therefore, in M1, the corrected voltage is higher than the real value. As for M2, $V_{O C 1}$, although varying with $T m$, is still closer to $V_{O C}^{\text {real }}$. Consequently, voltage correction is more efficient with M2. Regarding PS (1 module shaded), during the process of I-V scan, when the bypass diodes get activated, the shaded module can be considered as a SC. That explains why a similar deviation is also observed near the inflexion point.

From the above analysis, M1 and M2 have their pros and cons in voltage correction. Accordingly, NewM2 is designed by combining their advantages. As observed before, in $k_{2}$, using $V_{O C}^{S T C}$ (like in M1) could generally lead to better voltage correction than using $V_{O C 1}$ (like in M2) except under SC. The exception is because $V_{O C}^{S T C}$ fails to reflect $V_{O C}^{\text {real }}$. Therefore, in NewM2, $V_{O C 1}$ is replaced by $V_{O C 1} \cdot\left[1+\beta_{r e l}\left(T_{m 2}-T_{m 1}\right)\right]$, which corrects the measured $V_{O C 1}$ to the $V_{O C}$ under STC of the real curve and therefore, could better approximate $V_{O C}^{\text {real }}$ under any condition. In this way, as shown in Fig. 3 (d), the correction errors near the open-circuit point using M1 (under SC) and using M2 (under other cases) are attenuated.

\section{2) Current correction:}

For the current of corrected curves, noticeable dispersion along the $y$-axis is observed under PS near the inflexion point using M1. In fact, the output current of the PV module is mainly affected by $G$, while the impact of $T m$ is limited. Therefore, to analyze this phenomenon, for simplification, the contribution of ' $\left(T_{m 2}-T_{m 1}\right)$ ' is neglected. Then we can derive from (1) and (3):

- For M1:

$$
I_{2}=I_{1}+I_{S C 1}\left(G_{2} / G_{1}-1\right)
$$

- $\quad$ For M2:

$$
I_{2}=I_{1} \cdot G_{2} / G_{1}
$$

Comparing these two expressions, for M2, the corrected current $\left(I_{2}\right)$ is proportional to the current to correct $\left(I_{1}\right)$. While for M1, there is always a bias that degrades correction's performance, particularly around the inflexion point, like in Fig. 3 (b) under PS. Therefore, M2 performs relatively better than M1 in current correction. That also explains why, for NewM2, the current formula of M2 is chosen, and the same performance is achieved in Fig. 3 (d).

\subsection{2. $C E$ and $R E$ of key curve parameters}

Based on the corrected curves, the performance evaluation metrics $\left(C E, R E_{P_{m}}, R E_{V_{O C}}\right.$ and $\left.R E_{I_{S C}}\right)$ are calculated. Considering that $G$ and $T m$ are both varying for the 174 curves to correct, the statistics of the metrics are presented in Fig. 4.

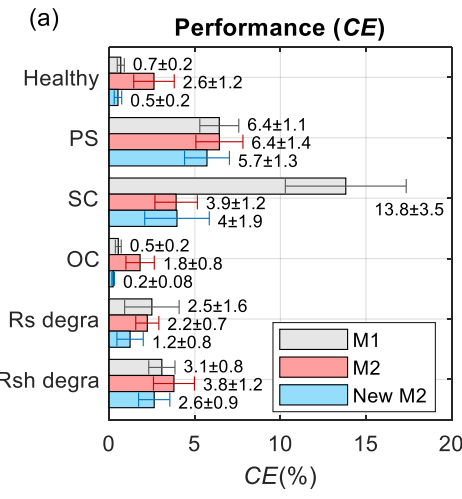

(b) Performance $\left(R E_{P m}\right)$

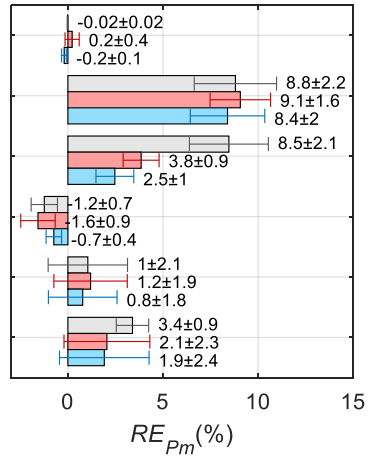

(c) Performance $\left(R E_{V o c}\right)$

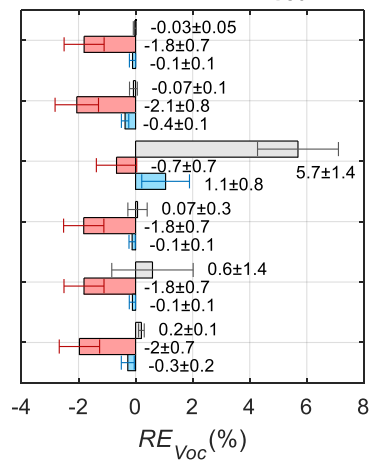

(d) Performance $\left(R E_{\text {Isc }}\right)$

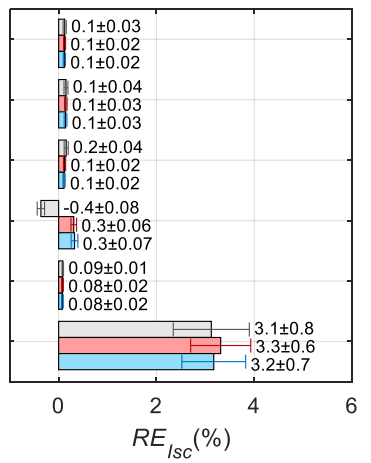

Fig. 4 Four metrics to present the correction performance of M1, M2 and NewM2: (a) $C E$, (b) $R E$ of $P_{m}$, (c) $R E$ of $V_{o c}$, (d) $R E$ of $I_{S C}$ (the bars represent the mean value for the correction of 174 curves, while the horizontal whiskers represent the standard deviation (std), these 2 values are marked as 'mean' \pm 'std')

These metrics are analyzed as follows: 
1) $C E$ : The value of $C E$, which reflects the correction error on the whole curve, corresponds to the observations in Fig. 3. As expected, high $C E$ is found under SC using M1, and PS using all the methods. None of M1 and M2 outperforms in all the conditions, but the proposed NewM2 performs better with generally lower and more stable $C E$. The overall average $C E(2.37 \%)$ decreased by $31.3 \%$ compared to the average when using M2 $(3.45 \%)$;

2) $R E_{P_{m}}$ : Similarly, NewM2 performs better correction of $P_{m}$. However, it should be noticed that $R E_{P_{m}}$ is positive under nearly all the conditions for all the methods. This indicates that the fault impact on $P_{m}$ is underestimated. The mean maximum value is up to $9.1 \%$, which could hinder the detection of incipient PV fault if $P_{m}$ is used as a fault indicator;

3) $R E_{V_{O C}}$ : The results are consistent with our previous observations, i.e., the corrected $V_{O C}$ with $\mathrm{M} 2$ is always lower than the real value under all conditions, while the $V_{O C}$ using $M 1$ is relatively better corrected except under $\mathrm{SC}$, where the maximum value of the mean $R E_{V_{O C}}$ could reach $5.7 \%$. And NewM2 effectively reduces $R E_{V_{O C}}$ compared with M1 (in SC), and M2 (in other cases);

4) $R E_{I_{S C}}$ : The performance of the three methods is similar. It should be noted that, based on (2) and (4), the voltage of the short-circuit point on the original curve (i.e., equals 0), after correction, is positive. This phenomenon is also observed in (Bühler et al., 2014). Therefore, to retrieve the $I_{S C}$ (according to the definition, the corresponding voltage should be 0 ), the corrected curve must be extrapolated. Here, the extrapolation method in (Bühler et al., 2014) is adopted. In this way, although the current value corrected from the original $I_{S C}\left(I_{S C 1}\right)$ is close to the actual $I_{S C}$, there is still a difference that depends on the initial slope of the corrected curve. Indeed for all the conditions except Rsh degradation, where the slope is mild, $R E_{I_{S C}}$ is low (within $\pm 0.5 \%$ ). But for Rsh degradation, where the current at MPP is severely lowered down, the slope is steep, and $R E_{I S C}$ is larger.

\subsection{3. $R E$ of fault features}

Now, we evaluate the correction performance from one or two typical curve parameters, selected as follows:

1) PS: The voltage and current at the inflexion point, named $V_{r p}$ and $I_{r p}$ respectively. $I_{r p}$ could reflect the shading level, and $V_{r p}$ the number of activated bypass diodes of PV modules in one string;

2) SC: The open-circuit voltage $V_{O C}$;

3) OC: The short-circuit current $I_{S C}$;

4) Rs degradation: The extracted $R_{S}$ from I-V curve, $R_{S}=-\frac{1}{\left.\frac{d l}{d V}\right|_{V=V_{O C}}}$ (Chin et al., 2015);

5) Rsh degradation: The extracted $R_{S h}$ from I-V curve, $R_{S h}=-\frac{1}{\left.\frac{d I}{d V}\right|_{I=I_{S C}}}$ (Chin et al., 2015);

For each fault feature, the $R E$ is calculated from the values obtained from the corrected and real curves. The results are summarized in Table 4. We can observe large misestimation (absolute mean $R E>2 \%$ ) with $V_{r p}, I_{r p}, V_{O C}$ and $R_{S}$ when using M1, and $V_{r p}, R_{s}$ and $R_{s h}$ when using M2 and NewM2. Worse yet, nearly all these large errors (except $R_{s h}$ using M2 and NewM2) lead to underestimating fault features, which will affect the detection of incipient faults. Similarly, none of the three methods outperforms for all the fault features. However, the overall performance of NewM2 is relatively better.

Table 4 Impact of correction on fault features using M1, M2 and NewM2

\begin{tabular}{|c|c|c|c|c|c|c|c|}
\hline \multirow[b]{2}{*}{ Case } & \multirow{2}{*}{$\begin{array}{c}\text { Fault } \\
\text { feature }\end{array}$} & \multicolumn{2}{|c|}{ M1 } & \multicolumn{2}{|c|}{ M2 } & \multicolumn{2}{|c|}{ NewM2 } \\
\hline & & $\begin{array}{c}\text { Value }(\%) \\
(\text { mean } \pm \text { std })\end{array}$ & $\begin{array}{l}\text { Impact on } \\
\text { fault feature }\end{array}$ & $\begin{array}{c}\text { Value }(\%) \\
(\text { mean } \pm \text { std })\end{array}$ & $\begin{array}{l}\text { Impact on } \\
\text { fault feature }\end{array}$ & $\begin{array}{c}\text { Value }(\%) \\
(\text { mean } \pm \text { std })\end{array}$ & $\begin{array}{c}\text { Impact on } \\
\text { fault feature }\end{array}$ \\
\hline \multirow{2}{*}{ PS } & $R E_{V_{r p}}$ & $7.3 \pm 2.6$ & Underestimated & $6.8 \pm 1.3$ & Underestimated & $7.4 \pm 1.8$ & Underestimated \\
\hline & $R E_{I_{r p}}$ & $2.5 \pm 6.6$ & Underestimated & $-0.2 \pm 0.1$ & Overestimated & $-0.2 \pm 0.1$ & Overestimated \\
\hline $\mathrm{SC}$ & $R E_{V_{O C}}$ & $5.7 \pm 1.4$ & Underestimated & $-0.7 \pm 0.7$ & Overestimated & $1.1 \pm 0.8$ & Underestimated \\
\hline $\mathrm{OC}$ & $R E_{I_{S C}}$ & $-0.4 \pm 0.08$ & Overestimated & $0.3 \pm 0.06$ & Underestimated & $0.3 \pm 0.07$ & Underestimated \\
\hline Rs degra & $R E_{R_{S}}$ & $-3.4 \pm 0.7$ & Underestimated & $-13.6 \pm 5.1$ & Underestimated & $-5.5 \pm 5.3$ & Underestimated \\
\hline Rsh degra & $R E_{R_{S h}}$ & $-0.1 \pm 0.4$ & Overestimated & $-4.6 \pm 9.0$ & Overestimated & $-4.1 \pm 9.1$ & Overestimated \\
\hline
\end{tabular}

\subsection{Correction performance using curves for defects of variable severity}

In this subsection, the impact of each fault severity on correction performance is investigated. The severities of PS, Rs, and Rsh degradations are varied in the following ranges one at a time: GainPS $=0: 0.1: 1, R_{s_{-}}$degra $=10^{-5}: 0.2: 2 \Omega$ or $R_{\text {sh_degra }}$ 
$=10^{1: 1: 5} \Omega$. For each fault level, the curves are simulated for the selected 174 pairs of $G$ and $\operatorname{Tm}$ (presented in Section 3.1), and corrected using the three single-curve based methods.

\subsection{1. $C E$ and $R E$ of key curve parameters}

The performance metrics extracted from these corrected curves are now presented in Fig. 5 as a function of fault severity.

(a)

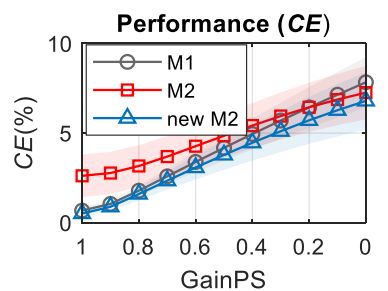

GainPS

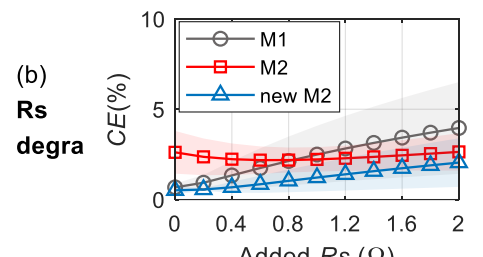

Added Rs $(\Omega)$

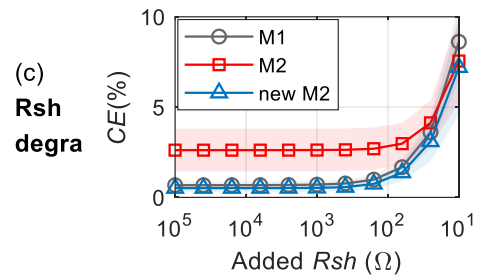

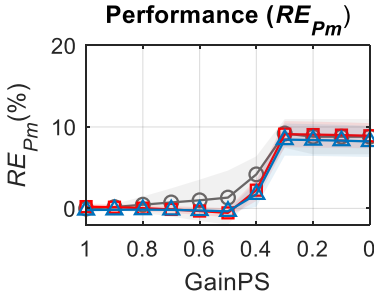

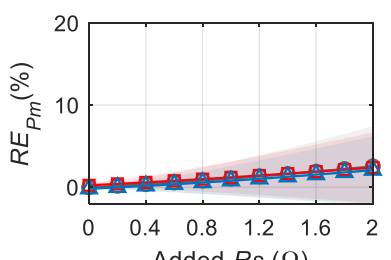

Added Rs $(\Omega)$

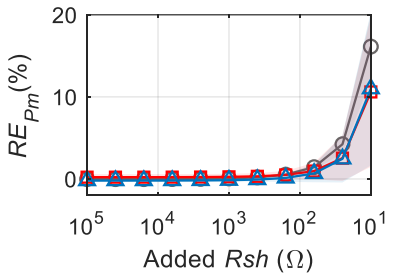

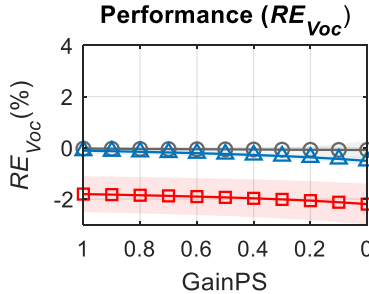
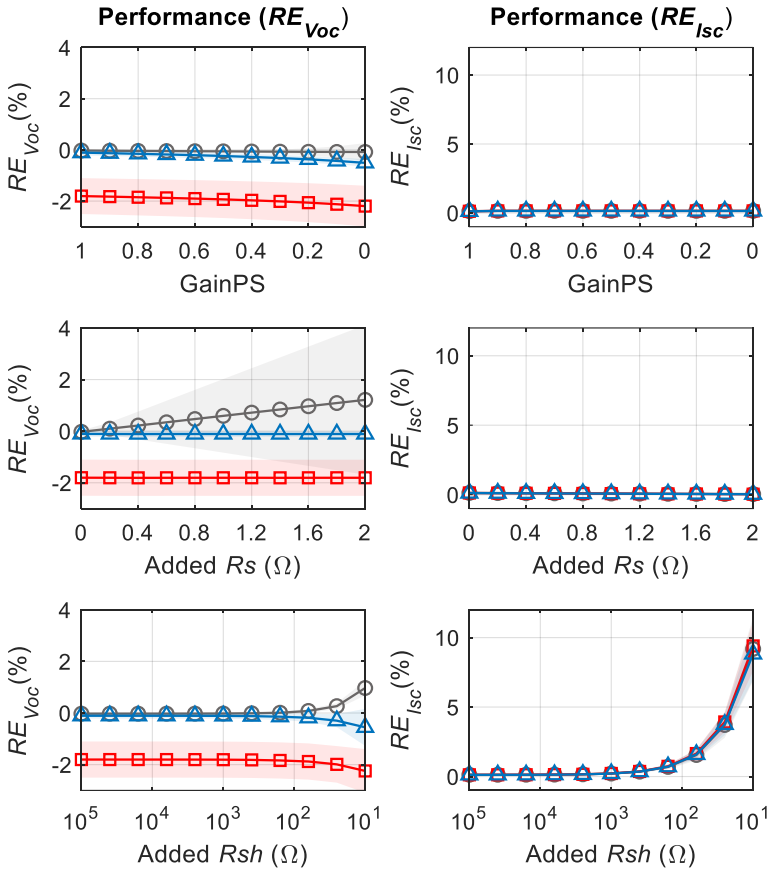

Fig. $5 C E$ and $R E$ of Pm, Voc and $I s c$ using M1, M2 and NewM2 under 3 faulty conditions with variable fault severity: (a) PS, (b) Rs degradation, (c) Rsh degradation (the marked line represents the mean of 174 values obtained from the correction of 174 under each fault severity, while the band area behind represents the standard deviation; the $x$-axis for Rsh degradation is set as log scale; the degree of severity is presented from healthy to severe on the $x$-axis from left to right)

From Fig. 5, several remarks can be drawn:

- All the metrics have a monotonic variation with the fault severities;

- $C E$ is mainly sensitive to PS and severe Rsh degradation;

- $R E_{P_{m}}$ is more sensitive to severe Rsh degradation and PS. The step variation observed for PS is due to the maximum power point (MPP) shifting as it can be observed in Fig. 6;

- $R E_{V_{O C}}$ and $R E_{I_{S C}}$ are almost insensitive to all faults regardless of their level of severity, except for $R E_{I_{S C}}$ under severe Rs degradation;

- The overall performance of NewM2 is better than M1 and M2 with relatively lower $C E$ and $R E$ for key curve parameters. 

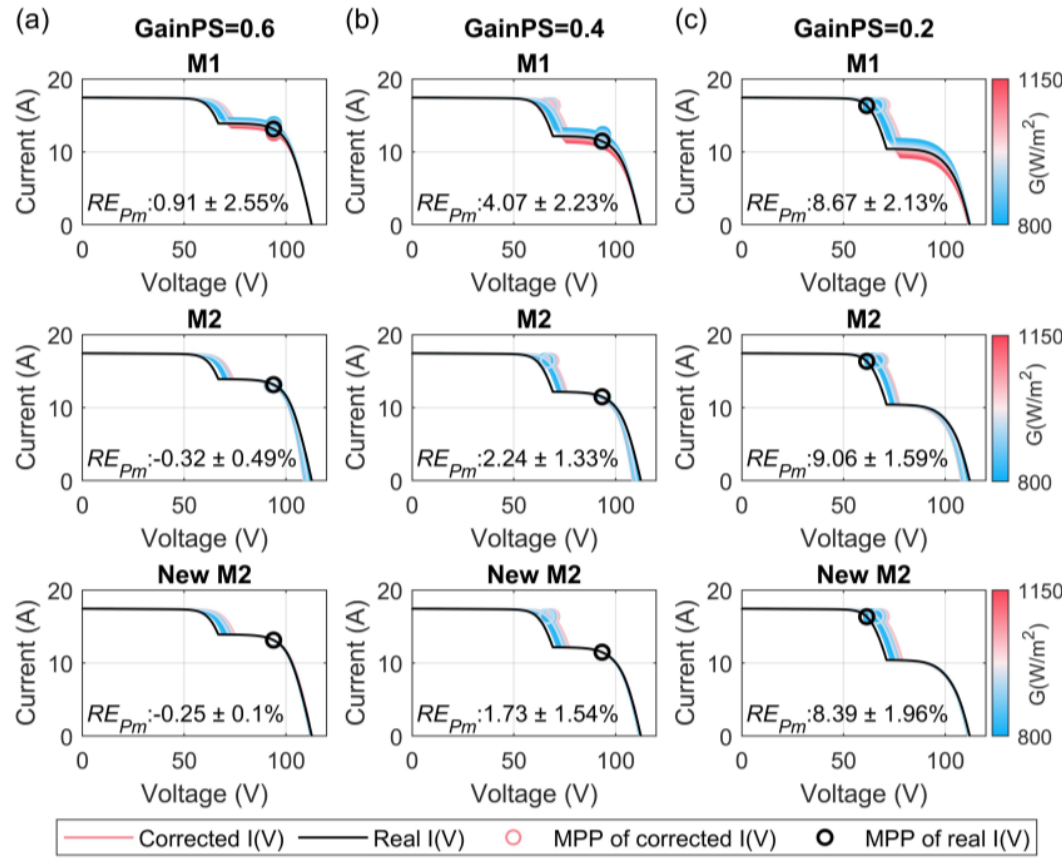

Fig. 6 Corrected curves and $R E_{P_{m}}$ using M1, M2 and NewM2 under three fault severities of PS: (a) GainPS = 0.6, (b) GainPS = 0.4, (c) GainPS $=0.2$ (the displayed color of corrected curves and MPP depends on the $G$ of the curve to correct, the circles on the curves represent the MPP, $R E_{P_{m}}$ are marked as 'mean' \pm 'std')

Moreover, it should be noted that, for $P_{m}$, which serves as an essential indicator for PV devices' health status, its $R E$ is always positive and gradually increases with the fault severity. This means that using M1 or M2 leads to an inevitable underestimation of the fault impact, which increases with the fault severity.

\subsection{2. $R E$ of fault features}

The impact of fault severity on fault features is also investigated, as illustrated in Fig. 7.
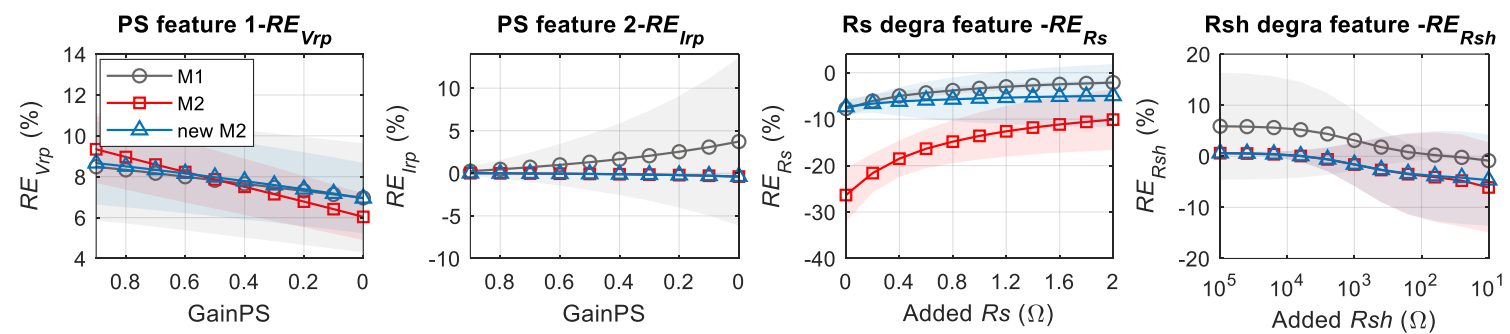

Fig. $7 R E$ of fault features under PS, Rs and Rsh degradations with variable fault severity (the marked line represents the mean of 174 values obtained from the correction of 174 curves under each fault severity, while the band area behind represents the standard deviation; the $x$-axis for Rsh degradation is set as log scale; the degree of severity is presented from healthy (except for $V_{r p}$ and $I_{r p}$ as these features do not exist under healthy condition) to severe on the $x$-axis from left to right)

Concerning the variation of the defect characteristics, different trends are observed: for the features $V_{r p}$ and $R_{s}$ using all the three methods and for $R_{s h}$ using M1, the $R E$ decreases as the severity of the defect increases. The opposite trend is observed for the other cases. This is due to the different changing rates of each feature's absolute error and its reference value, as defined in (12). Overall, NewM2 performs relatively better with lower and more stable $R E$.

It should be noted that within the range of severity of the defects tested; all characteristics are underestimated with the three methods. Therefore, used as defect signatures $\left(R_{s}\right.$ or $R_{s h}$, for example), which is typical for PV module degradation analyses, this could hamper the detection and diagnosis of these defects.

\section{Performance of correction methods using multiple I-V curves}

In this section, the method based on multiple curves (M3) will be evaluated. Section 4.1 presents the selection of $G$ and $T m$ for the reference curves. Sections 4.2 and 4.3 present the correction performance using simulated curves with the selected $G$ and $T m$, for constant and then variable fault severity. 


\subsection{Selection of $G$ and $T m$ for reference curves}

Different from M1 and M2, M3 needs at least three reference curves to complete one correction to STC. In this study, like other common applications of M3, we apply the 2-step correction methodology proposed in (IEC 60891, 2009) with three curves. An illustration of this methodology on $G-T m$ plot is shown in Fig. 8.

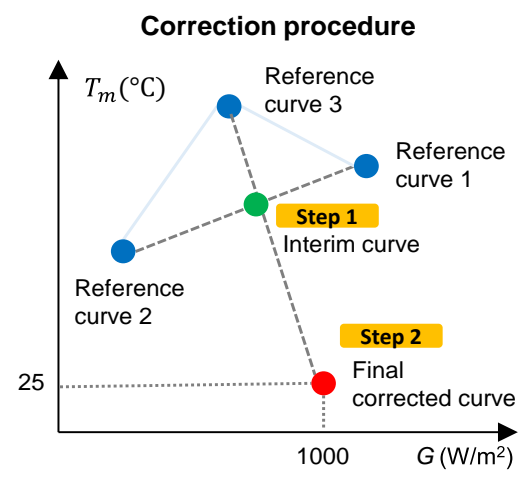

Fig. 8 Illustration of 2-step correction methodology based on three curves (Step 1: use reference curves 1 and 2 to generate the interim curve; Step 2: use reference curve 3 and the interim curve to get the final corrected curve)

Before the simulation of these curves, $G$ and $T m$ need to be determined. The field-measured distributions of $G$ and $T m$ of the same mc-Si module employed in the simulation represent as many real situations as possible. As shown in Fig. 8, three reference curves form one group of curves to get one corrected-to-STC curve. The irradiance $G$ and temperature $T m$ of these reference curves are determined by one-day measurement, covering sunny and cloudy conditions. The latter have larger fluctuations of $T m$. Besides, for sunny and cloudy conditions, three possible conditions are also considered for the irradiance: 1) lower than $1000 \mathrm{~W} / \mathrm{m}^{2}$, 2) around $1000 \mathrm{~W} / \mathrm{m}^{2}$ and 3) higher than $1000 \mathrm{~W} / \mathrm{m}^{2}$. In total, 120 groups of $G$ and Tm are manually selected. Some examples are shown in Fig. 9.
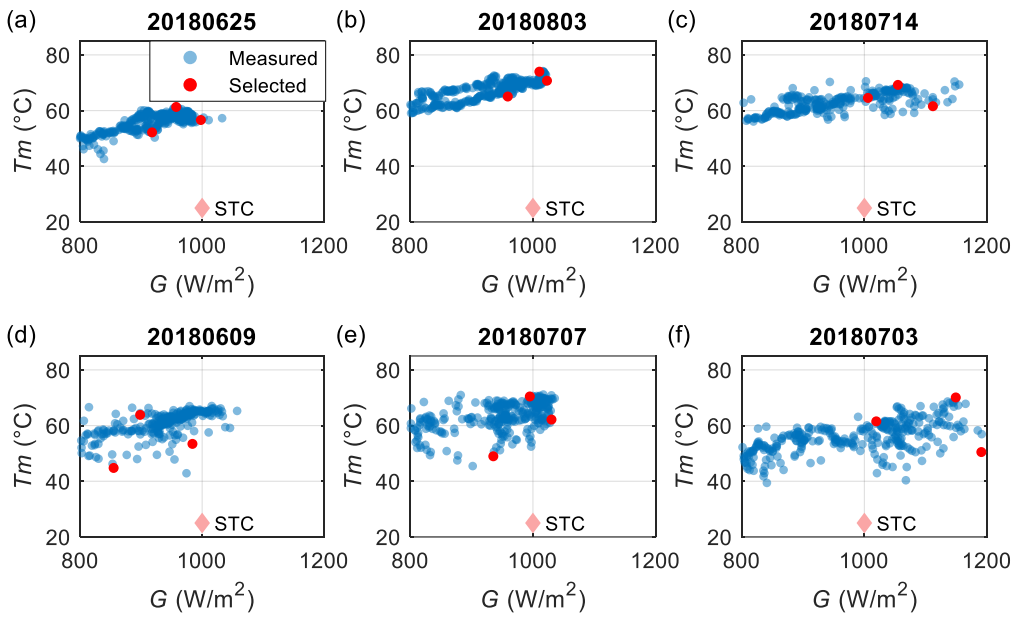

Fig. 9 Examples of selected $G$ and $T m$ from different summer days: (a, b, c) clear sunny days, (d, e, f) cloudy days ((a, d): the 3 selected $G<1000 \mathrm{~W} / \mathrm{m}^{2}$, (b, e): selected $G$ around $1000 \mathrm{~W} / \mathrm{m}^{2}$, (c, f): selected $G>1000 \mathrm{~W} / \mathrm{m}^{2}$ )

\subsection{Correction performance using field-measured $G$ and $T m$ for defect of constant fault severity}

Firstly, we use the same fault configuration presented in Section 3.1 and simulate 120 groups of reference curves regarding the fault severity setting. Then, M3 is applied to obtain 120 corrected curves, as shown in Fig. 10. The corresponding performance metrics and fault features are summarized in Table 5. 

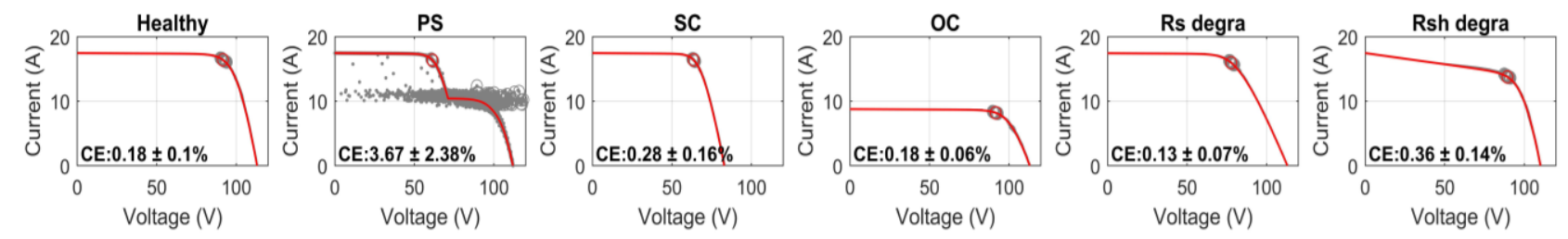

- Corrected I-V $\longrightarrow$ Real I-V

Voltage (V)

Fig. 10 Corrected curves using M3 (for PS, due to the large fluctuations, the corrected curves are plotted with dot line for better presentation)

Table 4 Four metrics to present the correction performance and the impact of correction on fault features using M3

\begin{tabular}{|c|c|c|c|c|c|c|c|}
\hline \multirow{2}{*}{ Case } & \multirow{2}{*}{$\begin{array}{c}\mathrm{CE}(\%) \\
(\text { mean } \pm \text { std })\end{array}$} & \multirow{2}{*}{$\begin{array}{c}R E_{P_{m}}(\%) \\
(\text { mean } \pm \text { std) }\end{array}$} & \multirow{2}{*}{$\begin{array}{c}R E_{V_{O C}}(\%) \\
(\text { mean } \pm \text { std) }\end{array}$} & \multirow{2}{*}{$\begin{array}{c}R E_{I_{S C}}(\%) \\
(\text { mean } \pm \text { std) }\end{array}$} & \multicolumn{3}{|c|}{ Fault feature } \\
\hline & & & & & Feature & Value $(\%)($ mean \pm std) & Impact on fault feature \\
\hline Healthy & $0.18 \pm 0.10$ & $-0.02 \pm 0.1$ & $0.07 \pm 0.04$ & $6.10^{-3} \pm 0.05$ & - & - & - \\
\hline \multirow{2}{*}{ PS } & \multirow{2}{*}{$3.67 \pm 2.38$} & \multirow{2}{*}{$7.5 \pm 18.3$} & \multirow{2}{*}{$0.06 \pm 0.07$} & \multirow{2}{*}{$6.10^{-3} \pm 0.05$} & $R E_{V_{r p}}$ & $-6.9 \pm 32.0$ & Overestimated \\
\hline & & & & & $R E_{I_{r p}}$ & $3.2 \pm 12.2$ & Underestimated \\
\hline $\mathrm{SC}$ & $0.28 \pm 0.16$ & $0.03 \pm 0.3$ & $0.2 \pm 0.1$ & $6.10^{-3} \pm 0.05$ & $R E_{V_{O C}}$ & $0.2 \pm 0.1$ & Underestimated \\
\hline OC & $0.18 \pm 0.06$ & $2 \mathrm{e}-4 \pm 0.2$ & $0.07 \pm 0.05$ & $6.10^{-3} \pm 0.05$ & $R E_{I_{S C}}$ & $6.10^{-3} \pm 0.05$ & Underestimated \\
\hline Rs degra & $0.13 \pm 0.07$ & $0.1 \pm 0.2$ & $0.07 \pm 0.03$ & $6.10^{-3} \pm 0.05$ & $R E_{R_{S}}$ & $0.01 \pm 0.04$ & Overestimated \\
\hline Rsh degra & $0.36 \pm 0.14$ & $0.4 \pm 0.2$ & $0.05 \pm 0.04$ & $6.10^{-3} \pm 0.05$ & $R E_{R_{S h}}$ & $4.10^{-3} \pm 0.01$ & Underestimated \\
\hline
\end{tabular}

From the results, clearly, except for PS, M3 achieves good correction (mean value of all metrics within $\pm 1 \%$ ) for all the conditions.

In the following, we analyze the 'catastrophic' correction error near the inflexion point under PS. As illustrated in Fig. 8, the correction procedure using M3 is done in two steps. An example of these steps under PS is shown in Fig. 11. In step 1, due to the unsynchronized appearance of inflexion points on reference curves 1 and 2 (i.e., $V_{r p}$ are not identical), another inflexion point is created on the intermediate curve, so that in step 2, a strange curve shape is introduced. This phenomenon could lead to a large error on $P_{m}$ but with no significant impact on $V_{o c}$ and $I_{S C}$.
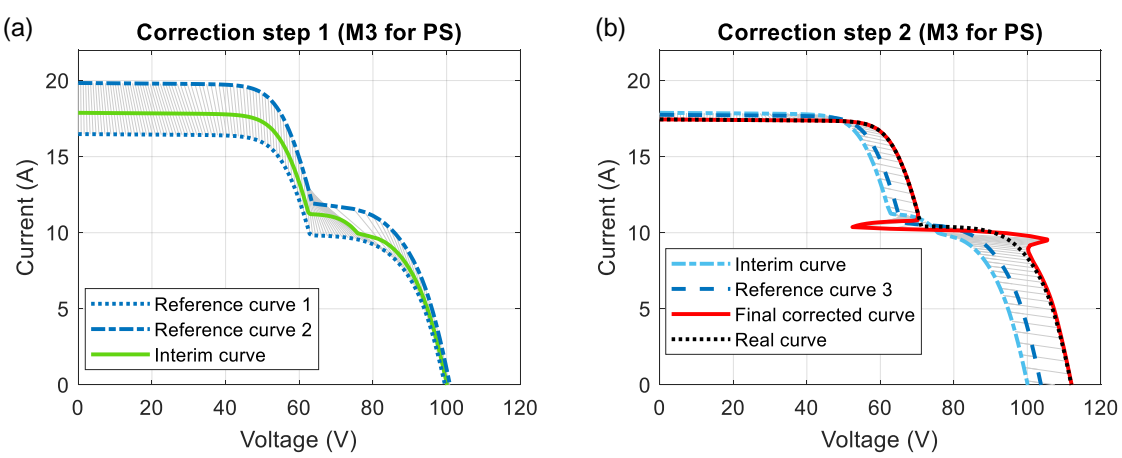

Fig. 11 Correction procedure under PS using 3 reference curves-based M3: (a) correction step 1, (b) correction step 2

Also, it should be noted that the fault impact on all the fault features is underestimated. Furthermore, as expected, the most significant error occurs under PS. Under the other conditions, the $R E$ is within $\pm 1 \%$.

\subsection{Correction performance using field-measured $G$ and $T m$ for defects of variable severity}

In this subsection, the impact of fault severity on correction performance using M3 is investigated. The severity for PS, Rs and Rsh degradations is varied using the same setting as in Section 3.2. The performance metrics as a function of fault severity are presented in Fig. 12. 

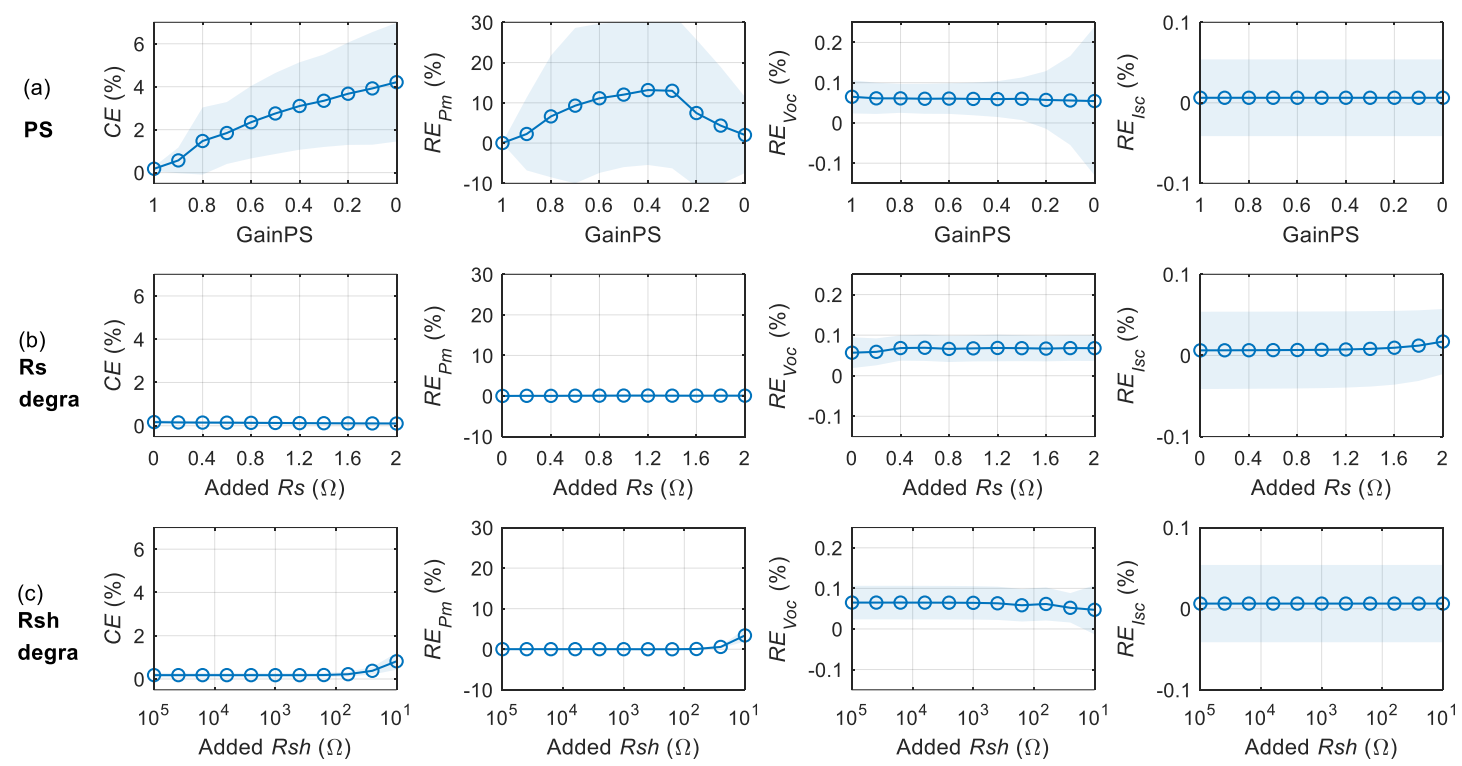

Fig. $12 C E$ and $R E$ of $P m, V o c$ and $I s c$ for M3 under 3 faulty cases with variable fault severity: (a) PS, (b) Rs degradation, (c) Rsh degradation (the circled line represents the mean value for the 120 corrected curves for each fault severity, while the band area behind represents the standard deviation; the $x$-axis for Rsh degradation is set as log scale; the degree of severity is presented from healthy to severe on the $x$-axis from left to right)

From these results, the following remarks could be drawn:

- Regarding Rs and Rsh degradations, all the metrics are insensitive to the fault level;

- Regarding PS, only $C E$ and $R E_{P m}$ vary according to the fault severity. $C E$ has a monotonic variation, which makes it relevant for fault severity estimation. However, $R E_{P m}$ is not a reliable feature as it exhibits a nonmonotonic variation. This phenomenon is due to the displacement of the maximum power point (MPP) as illustrated in Fig. 13.
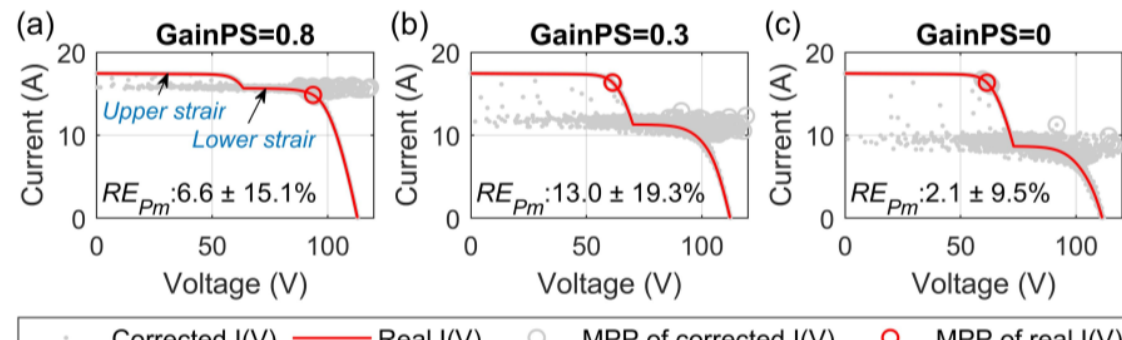

$$
\text { Corrected I(V) } \longrightarrow \text { Real I(V) } \bigcirc \text { MPP of corrected I(V) }
$$

MPP of real I(V)

Fig. 13 Corrected curves using M3 under 3 fault severities of PS: (a) GainPS=0.8, where MPP of real and corrected curves are all at 'lower stair', (b) GainPS = 0.3, where MPP of real is at 'upper stair' while MPP of most corrected curves at 'lower stair' (c) GainPS = 0 , MPP of real and most corrected curves are all at 'upper stair' (for each case, $R E_{P_{m}}$ is marked as 'mean' \pm 'std')

The behavior of fault features is also investigated with the comparison of identified features from both corrected and real I-V curves presented in Fig. 14 and the corresponding relative errors $(R E)$ in Fig. 15. 

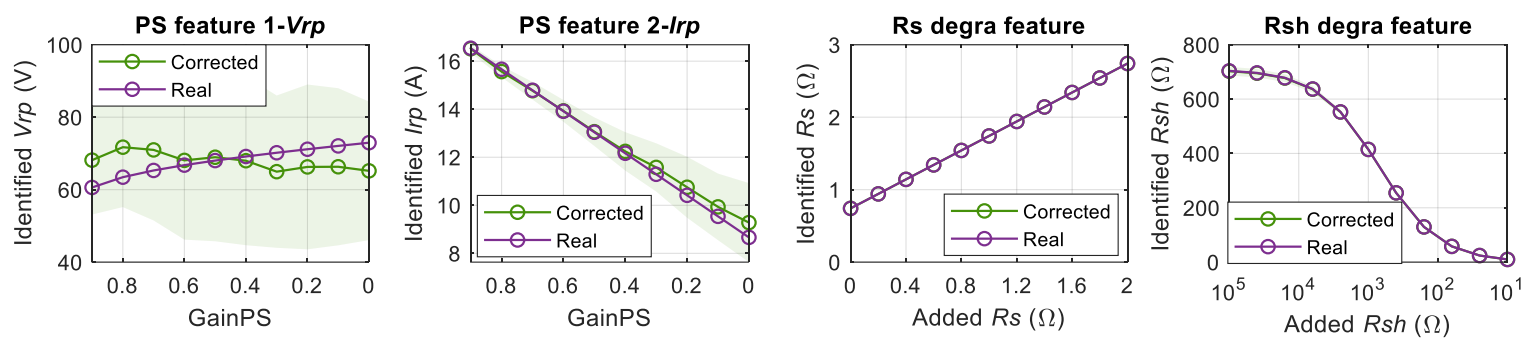

Fig. 14 Identified feature from corrected (using M3) and original I-V curve for fault of variable severity (the circled line represents the mean, while the band area represents the standard deviation; the values of $R_{S}$ or $R_{s h}$ identified from I-V curve are not equal to the additional resistance (illustrated in Fig. 1) due to the existence of inherent equivalent $R_{S}(0.74 \Omega)$ or $R_{s h}(708.36 \Omega)$ of PV arrays
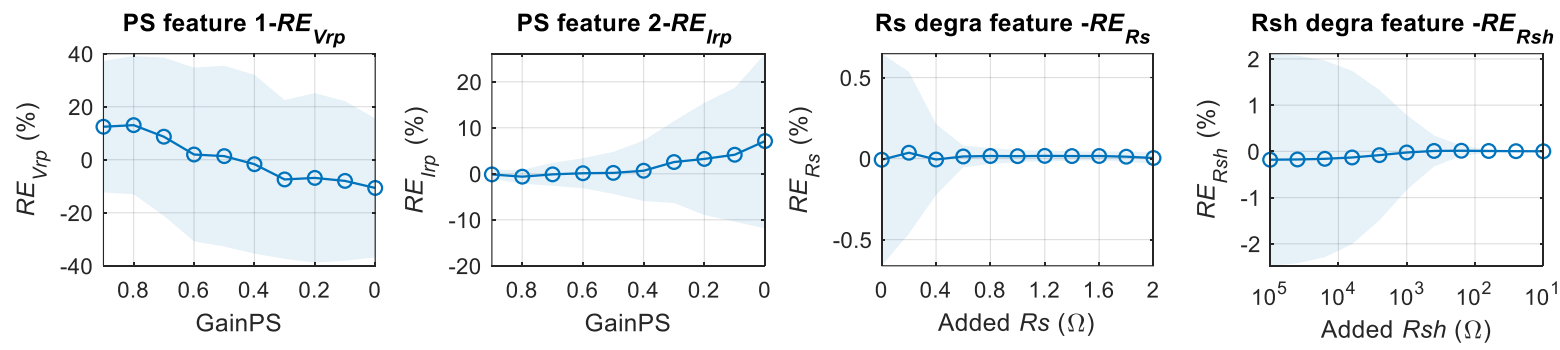

Fig. $15 R E$ of fault features under PS, Rs and Rsh degradations for fault of variable severity using M3 (the circled line represents the mean, while the band area represents the standard deviation)

The large dispersion observed for $R E_{V r p}$ and $R E_{I r p}$ confirms the poor performance of the correction near the inflection point. The values of $R_{S}$ and $R_{S h}$ extracted from the original and corrected curves are very close, as confirmed in Fig. 14. This is also reflected in the low values of $R E_{R_{S}}$ and $R E_{R_{S h}}$ displayed in Fig. 15. The mean value of $R E_{R_{S}}$ lies within $\pm 0.1 \%$ and that of $R E_{R_{s h}}$ within $\pm 0.2 \%$, and both are relatively insensible to the varying fault severity. It is also noted that the standard deviations of $R E_{R_{S}}$ and $R E_{R_{S h}}$ exhibit a decreasing trend when the fault severity increases. In all, with varying fault, M3 achieves quite good and robust correction under Rs and Rsh degradation.

\section{Discussion}

\subsection{Summary of correction methods}

The correction performance using three single and one multiple curves-based methods has been evaluated. Each method has its own pros and cons listed in Table 6.

Table 6 Pros and Cons of correction methods

\begin{tabular}{|c|c|c|c|c|}
\hline & \multicolumn{3}{|c|}{ Single curve-based methods } & \multirow[t]{2}{*}{ Multiple curves-based method (M3) } \\
\hline & M1 & M2 & NewM2 & \\
\hline Pros & $\begin{array}{l}\text { - Better correction of } \\
\text { voltage than M2 } \\
\text { - Suitable for rapid fie }\end{array}$ & $\begin{array}{l}\text { - Better correction of } \\
\text { current than M1 } \\
\text { agnosis }\end{array}$ & $\begin{array}{l}\text { - Better overall performance } \\
\text { than M1 and M2 }\end{array}$ & $\begin{array}{l}\text { - High-precision correction performance under } \\
\text { most conditions } \\
\text { - Free of correction coefficients }\end{array}$ \\
\hline Cons & $\begin{array}{l}\text { - Needs to determine } t \\
\text { - Prone to large under }\end{array}$ & $\begin{array}{l}\text { orrection coefficients } \\
\text { lation of } P_{m} \text {, and fault } \mathrm{fe}\end{array}$ & & $\begin{array}{l}\text { - Not suitable for rapid field diagnosis } \\
\text { - Needs at least } 3 \text { well-chosen reference curves } \\
\text { - Poor correction near inflexion point under PS } \\
\text { with high effect on } P_{m}\end{array}$ \\
\hline
\end{tabular}

Once established (i.e., correction coefficients determined), all these single curve-based correction methods can conduct rapid correction of measured I-V curves. This allows their integration in real-time health monitoring of PV devices. However, as discussed in Section 2.2, the determination of correction coefficients is a troublesome task. The coefficients determined using simulated data do not always fit the real values, especially for the installed PV panels after a long-time operation. The proposed NewM2, which combines M1 and M2's advantages, leads to better correction performance in all 
fault conditions tested. However, it still introduces a significant correction error in the case of partial shading (PS), the degradation of Rs and Rsh, compared to healthy conditions, short circuit (SC) and open circuit (OC).

As for the multiple-curves-based method (M3), except under PS, excellent correction is obtained. However, the selection of reference curves is still a manual process (Hishikawa et al., 2016; IEC 60891, 2009), which could hinder its application in rapid or on-line field correction.

\subsection{Suggestions for future work}

Based on comparing the three IEC 60891 correction methods and the new proposal, some suggestions on future work are hereafter presented.

For correction methods based on a single curve, the determination of correction coefficients for PV panels on-site remains difficult. A strategy for determining these coefficients based on field measured data needs to be developed that differs from the IEC 60891 procedure, which requires environmental conditions that are only practically feasible in fully equipped laboratories. Solutions to reduce dependence on correction coefficients, such as (Hishikawa et al., 2019, 2018), should also be further developed and validated with I-V curves taken from panels with defects.

The proposed new correction method still needs to be evaluated with more types of defects.

For the correction method based on multiple curves, two improvements are expected. On the one hand, efforts are needed to improve correction performance in case of partial shading; on the other hand, developing a methodology for automatic and efficient selection of reference curves would facilitate the deployment of the technique in the field.

Correction methods should be evaluated with field-measured data.

\section{Conclusion}

In this work, we have evaluated the performance of methods based on one or more curves proposed in the IEC 60891 standard for the correction of I-V curves measured on defective photovoltaic panels. It has been shown that all the methods introduce significant errors due to irradiance, module temperature and the severity of the defects. Using the standard method M1 of the IEC, we have pointed out that a distortion of the curve's shape is commonly introduced with a relative error up to $13.8 \%$. For $P_{m}$ and the fault characteristics extracted from the corrected curves, estimation errors also occur frequently. Even worse still, most significant errors result in underestimating the characteristics (up to $9.1 \%$ for $P_{m}$ using M2). This can affect the detection of incipient PV defects if these characteristics are used as defect signatures.

Among the single curve-based methods (M1 and M2), none of them could outperform under all the faulty simulated conditions. Therefore, an M2-based improved method (NewM2) has been proposed. It has exhibited more robust overall performance than $\mathrm{M} 1$ and $\mathrm{M} 2$ with the decrease of average curve error from $3.45 \%$ to $2.37 \%$.

The method based on multiple curves (M3) generally has higher performance than those based on a single curve, but it is not suitable for rapid field diagnosis.

PV health monitoring and fault diagnosis using I-V curves is a promising approach. However, several signs of progress are expected from future work to improve its efficiency and ease its implementation. At first, the determination of correction coefficients based on field-measured data or mitigating the dependence on correction coefficients would be beneficial. Second, the development of a methodology for automatic determination of reference curves and improved correction performance (mainly to avoid underestimating incipient faults) under different faulty conditions.

\section{Acknowledgment}

The authors would like to acknowledge the SIRTA for the field-measured data and the China Scholarship Council (CSC) for the $\mathrm{PhD}$ funding.

\section{References}

Agrawal, N., Bora, B., Kapoor, A., 2020. Experimental investigations of fault tolerance due to shading in photovoltaic modules with different interconnected solar cell networks. Sol. Energy 211, 1239-1254. https://doi.org/10.1016/j.solener.2020.10.060

Bouaichi, A., Alami, A., Hajjaj, C., Messaoudi, C., Ghennioui, A., Benlarabi, A., Ikken, B., El, A., Zitouni, H., 2019. Insitu evaluation of the early PV module degradation of various technologies under harsh climatic conditions : The case of Morocco. Renew. Energy 143, 1500-1518. https://doi.org/10.1016/j.renene.2019.05.091 
Bühler, A.J., Perin Gasparin, F., Krenzinger, A., 2014. Post-processing data of measured I-V curves of photovoltaic devices. Renew. Energy 68, 602-610. https://doi.org/10.1016/j.renene.2014.02.048

Chen, Z., Wu, L., Cheng, S., Lin, P., Wu, Y., Lin, W., 2017. Intelligent fault diagnosis of photovoltaic arrays based on optimized kernel extreme learning machine and I-V characteristics. Appl. Energy 204, 912-931. https://doi.org/10.1016/j.apenergy.2017.05.034

Chin, V.J., Salam, Z., Ishaque, K., 2015. Cell modelling and model parameters estimation techniques for photovoltaic simulator application: A review. Appl. Energy 154, 500-519. https://doi.org/10.1016/j.apenergy.2015.05.035

Ding, K., Zhang, J., Bian, X., Xu, J., 2014. A simplified model for photovoltaic modules based on improved translation equations. Sol. Energy 101, 40-52. https://doi.org/10.1016/j.solener.2013.12.016

Dolara, A., Lazaroiu, G.C., Leva, S., Manzolini, G., 2013. Experimental investigation of partial shading scenarios on PV (photovoltaic) modules. Energy 55, 466-475. https://doi.org/10.1016/j.energy.2013.04.009

Fadhel, S., Delpha, C., Diallo, D., Bahri, I., Migan, A., Trabelsi, M., Mimouni, M.F., 2019. PV shading fault detection and classification based on I-V curve using principal component analysis: Application to isolated PV system. Sol. Energy 179, 1-10. https://doi.org/10.1016/j.solener.2018.12.048

Friesen, G., Herrmann, W., Belluardo, G., Herteleer, B., 2018. Report IEA-PVPS T13-11:2018 Photovoltaic module energy yield measurements: Existing approaches and best practice, IEA-PVPS. URL https://iea-pvps.org/wpcontent/uploads/2020/01/Photovoltaic_Module_Energy_Yield_Measurements_Existing_Approaches_and_Best_Pr actice_by_Task_13.pdf (accessed 6.15.20).

Golive, Y.R., Singh, H.K., Kottantharayil, A., Vasi, J., Shiradkar, N., 2019. Investigation of Accuracy of various STC Correction Procedures for I-V Characteristics of PV Modules Measured at Different Temperature and Irradiances, in: 2019 IEEE 46th Photovoltaic Specialists Conference (PVSC). IEEE, Chicago, IL, USA, pp. 2743-2748. https://doi.org/10.1109/PVSC40753.2019.8980557

Hishikawa, Y., Doi, T., Higa, M., Yamagoe, K., Ohshima, H., Takenouchi, T., Yoshita, M., 2018. Voltage-dependent temperature coefficient of the I-V curves of crystalline silicon photovoltaic modules. IEEE J. Photovoltaics 8, 4853. https://doi.org/10.1109/JPHOTOV.2017.2766529

Hishikawa, Y., Ohshima, H., Higa, M., Yamagoe, K., Doi, T., 2016. Precise Determination of the STC I-V Curves by Wide-Range Linear Extrapolation of Outdoor I-V Curves on Partly Sunny Days, in: 32nd European Photovoltaic Solar Energy Conference and Exhibition (Eu-PVSEC). München, Germany, pp. 1716-1719. https://doi.org/10.4229/EUPVSEC20162016-5DO.11.4

Hishikawa, Y., Takenouchi, T., Higa, M., Yamagoe, K., Ohshima, H., Yoshita, M., 2019. Translation of Solar Cell Performance for Irradiance and Temperature from a Single I-V Curve Without Advance Information of Translation Parameters. IEEE J. Photovoltaics 9, 1195-1201. https://doi.org/10.1109/JPHOTOV.2019.2924388

Huang, J.M., Wai, R.J., Gao, W., 2019. Newly-designed fault diagnostic method for solar photovoltaic generation system based on IV-Curve measurement. IEEE Access 7, 70919-70932. https://doi.org/10.1109/ACCESS.2019.2919337

Huawei, 2020. Smart I-V Curve Diagnosis. URL https://solar.huawei.com/enGB/download?p=\%2F-\%2Fmedia\%2FSolar\%2Fattachment $\% 2 F p d f \% 2 F e u \% 2 F d a t a s h e e t \% 2 F I V-C u r v e . p d f$ (accessed 8.17.20).

IEC 60891, 2009. Photovoltaic devices - Procedures for temperature and irradiance corrections to measured I-V characteristics.

Li, Y., Ding, K., Zhang, J., Chen, F., Chen, X., Wu, J., 2019. A fault diagnosis method for photovoltaic arrays based on fault parameters identification. Renew. Energy 143, 52-63. https://doi.org/10.1016/j.renene.2019.04.147

Ma, M., Liu, H., Zhang, Z., Yun, P., Liu, F., 2019. Rapid diagnosis of hot spot failure of crystalline silicon PV module based on I-V curve. Microelectron. Reliab. 100-101. https://doi.org/10.1016/j.microrel.2019.113402

Martínez-Moreno, F., Figueiredo, G., Lorenzo, E., 2018. In-the-field PID related experiences. Sol. Energy Mater. Sol. 
Cells 174, 485-493. https://doi.org/10.1016/j.solmat.2017.09.037

Mellit, A., Tina, G.M., Kalogirou, S.A., 2018. Fault detection and diagnosis methods for photovoltaic systems: A review. Renew. Sustain. Energy Rev. 91, 1-17. https://doi.org/10.1016/j.rser.2018.03.062

Migan, A., Mambrini, T., Bourdin, V., Badosa, J., 2015. Deployment of a multi-technology photovoltaic module test bench on the SIRTA meteorological and climate observatory, in: 31st European PV Solar Energy Conference and Exhibition (Eu-PVSEC). Hamburg, Germany.

Pillai, D.S., Rajasekar, N., 2018. A comprehensive review on protection challenges and fault diagnosis in PV systems. Renew. Sustain. Energy Rev. 91, 18-40. https://doi.org/10.1016/j.rser.2018.03.082

Rajput, P., Tiwari, G.N., Sastry, O.S., Bora, B., Sharma, V., 2016. Degradation of mono-crystalline photovoltaic modules after 22 years of outdoor exposure in the composite climate of India. Sol. Energy 135, 786-795. https://doi.org/10.1016/j.solener.2016.06.047

Sarikh, S., Raoufi, M., Bennouna, A., Benlarabi, A., Ikken, B., 2020. Implementation of a plug and play I-V curve tracer dedicated to characterization and diagnosis of PV modules under real operating conditions. Energy Convers. Manag. 209. https://doi.org/10.1016/j.enconman.2020.112613

Silva, A.M., Melo, F.C., Reis, J.H., Freitas, L.C.G., 2019. The study and application of evaluation methods for photovoltaic modules under real operational conditions, in a region of the Brazilian Southeast. Renew. Energy 138, 1189-1204. https://doi.org/10.1016/j.renene.2019.01.129

Spataru, S., Sera, D., Kerekes, T., Teodorescu, R., 2015. Monitoring and Fault Detection in Photovoltaic Systems Based On Inverter Measured String I-V Curves, in: 31st European Photovoltaic Solar Energy Conference and Exhibition (EU PVSEC). Hamburg, Germany, pp. 1667-1674. https://doi.org/10.4229/EUPVSEC20152015-5BO.12.2

Tanesab, J., Parlevliet, D., Whale, J., Urmee, T., 2017. Seasonal effect of dust on the degradation of PV modules performance deployed in different climate areas. Renew. Energy 111, 105-115. https://doi.org/10.1016/j.renene.2017.03.091

Tanesab, J., Parlevliet, D., Whale, J., Urmee, T., Pryor, T., 2015. The contribution of dust to performance degradation of PV modules in a temperate climate zone. Sol. Energy 120, 147-157. https://doi.org/10.1016/j.solener.2015.06.052

Triki-Lahiani, A., Bennani-Ben Abdelghani, A., Slama-Belkhodja, I., 2018. Fault detection and monitoring systems for photovoltaic installations: A review. Renew. Sustain. Energy Rev. 82, 2680-2692. https://doi.org/10.1016/j.rser.2017.09.101

Wang, M., Liu, J., Burleyson, T.J., Schneller, E.J., Davis, K.O., French, R.H., Braid, J.L., 2020. Analytic

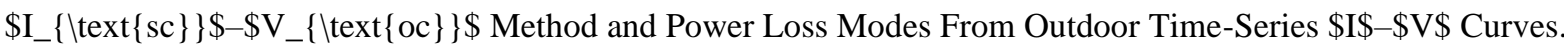
IEEE J. Photovoltaics 10, 1379-1388. https://doi.org/10.1109/JPHOTOV.2020.2993100

Zhu, H., Lu, L., Yao, J., Dai, S., Hu, Y., 2018. Fault diagnosis approach for photovoltaic arrays based on unsupervised sample clustering and probabilistic neural network model. Sol. Energy 176, 395-405. https://doi.org/10.1016/j.solener.2018.10.054

Zhu, Y., Xiao, W., 2020. A comprehensive review of topologies for photovoltaic I-V curve tracer. Sol. Energy. https://doi.org/10.1016/j.solener.2019.12.020 